\title{
Politique
}

\section{Étude du mouvement de l'opinion causé par le jugement de la Cour suprême sur l'avortement}

\author{
Jean H. Guay, Richard Nadeau et Édouard Cloutier
}

Numéro 15, hiver 1989

Paradigmes et scientificité

URI : https://id.erudit.org/iderudit/040619ar

DOI : https://doi.org/10.7202/040619ar

Aller au sommaire du numéro

Éditeur(s)

Société québécoise de science politique

ISSN

0711-608X (imprimé)

1918-6584 (numérique)

Découvrir la revue

Citer cet article

Guay, J. H., Nadeau, R. \& Cloutier, É. (1989). Étude du mouvement de l'opinion causé par le jugement de la Cour suprême sur l'avortement. Politique, (15),

53-77. https://doi.org/10.7202/040619ar d'utilisation que vous pouvez consulter en ligne. 


\title{
Étude du mouvement de l'opinion causé par le jugement de la Cour suprême sur l'avortement
}

\author{
Jean H. Guay, \\ Université d'Ottawa \\ Richard Nadeau, \\ Édouard Cloutier \\ Université de Montréal
}

Le 28 janvier 1988, la Cour suprême du Canada déclarait inconstitutionnelles toutes les dispositions du Code criminel régissant l'avortement. Cette transformation radicale du code légal ne pouvait manquer d'affecter l'opinion des citoyens à l'égard de la réglementation de l'avortement. La présente analyse tente de mesurer, à l'aide d'une démarche quasi-expérimentale, à quel rythme et de quelle manière les individus réorganisent les différentes composantes de leurs opinions suite à un jugement qui rend légal ce qui, jusque-là, était considéré illégal.

Notre analyse procédera en six étapes. Premièrement, nous passerons en revue les quelques écrits produits sur cette question. Deuxièmement, nous évaluerons les données présentement 
disponibles. Troisièmement, nous présenterons notre procédure d'enquête. Quatrièmement, nous décrirons le mouvement de l'opinion. Cinquièmement, nous expliquerons les facteurs de ce mouvement. En conclusion, nous discuterons d'une manière critique des résultats présentés.

\section{Un problème rarement posé}

Peu de chercheurs ont analysé l'influence exercée sur l'opinion publique par des jugements de cour. En fait, seulement quatre études peuvent être considérées comme pertinentes à ce sujet soit celles de Barnum (1985), de Baas et Thomas (1984), de Lehne et Reynolds (1978) et de Casey (1976).

Une première question étudiée se situe tout juste en amont de la nôtre: les opinions émises par la Cour suprême sont-elles convergentes avec les opinions majoritaires? Une première réponse provient d'une recherche faite par la maison Gallup. À partir de 13 jugements survenus entre 1954 et 1973, le rapport serait très ténu entre les décisions de la Cour suprême américaine et l'opinion publique: la Cour ne semble ni entériner, ni créer une majorité dans l'opinion (Gallup Poll Opinion Index, pages 11-13). Dans une récente étude Barnum a fourni une réponse plus nuancée en suggérant que la Cour s'appuie la plupart du temps soit sur une majorité, soit sur une minorité émergeante.

La recherche de Baas et Thomas, deuxièmement, remet en question une idée de Dahl qui avait soutenu en 1957: «The main task of the Court is to confer legitimacy on the fundamental policies of the successful coalition». Les auteurs essaient d'évaluer si la Cour se trouve effectivement à légitimer une position et, par là, à accroître sa popularité. Leur étude expérimentale, fondée sur des groupes d'étudiants universitaires, contredit Dahl. «The results of these three experiments are quite clear: with the exception of one out of 16 instances, mere endorsement by the Supreme Court alone, or the Court as interpreter of the Constitution, is not sufficient to elevate significantly mass acceptance of an issue or policy». Les auteurs soutiennent donc la position de Adamany selon lequel: «there can be 
no question that the widely asserted legitimacy-conferring function of the Supreme Court cannot summon adequate empirical support from public opinion studies...». Ils concluent: «Thus, the Court may in fact have a legitimating capacity that is manifested in behavioral compliance and not in policy supports.

Alors que les précédentes études cherchaient à cerner le lien causal direct entre une décision de la Cour et le mouvement de l'opinion, d'autres travaux se sont surtout penchés sur l'effet de variables intermédiaires interagissant sur ce processus. Dans cette série de travaux, deux types de variables ont été examinés. Le premier type concerne les caractéristiques des individus et dresse le profil des répondants les plus susceptibles d'être influencés par une décision de la Cour. À ce chapitre, les travaux de Lehne et Reynolds (1978) doivent être signalés puisqu'ils démontrent que l'idéologie des individus est peu affectée par les jugements de la Cour; chacun se forge une opinion davantage en fonction des réactions du Congrès ou du Président: «citizens evaluate their court primarily as a part of the institutional structure of the state government, partially as the source of a recent decision expressing specific policy conclusions, and not at all as a proponent of ideological or partisan viewpoints». En fait, les variables de l'ordre de l'identification partisane et de l'idéologie peuvent être considérées comme significatives uniquement pour les citoyens attentifs.

Le dernier aspect étudié renvoie à la nature et à l'impact de la couverture médiatique des décisions des tribunaux. Ainsi Casey (1976) distingue deux périodes. La première, qui suit immédiatement le jugement, se trouve à exagérer l'importance de la décision ou à la poser comme plus radicale qu'elle ne l'est; la seconde, au contraire, «soft pedaled the decision by presenting a limited, moderate view of it». Le grand public subirait de front la première des deux présentations médiatiques, tandis que l'élite assumerait la seconde. L'auteur se préoccupe donc beaucoup de la qualification des répondants dans son enquête: le grand public a moins d'aptitudes, selon cette étude, pour jouer avec les idées ou assumer les multiples positions qui émergent sur la scène publique. Les répondants du grand public subissent ainsi davantage la dissonance cognitive ou un déséquilibre entre l'estime qu'ils ont de la Cour et le jugement que celle-ci vient de rendre lorsqu'il 
est contradictoire avec leur opinion propre. À la limite, la Cour jouirait d'un plus grand appui des individus plus scolarisés, plus politisés et plus exposés aux informations politiques. L'attitude à l'endroit de la Cour est donc complexe.

Trois conclusions se dégagent des études sur l'impact des décisions de la Cour suprême sur le mouvement de l'opinion publique américaine: 1) l'effet de la Cour serait mitigé (présent dans certains cas, absent dans d'autres), circonscrit dans le temps et modeste quant à son ampleur; 2) certains individus seraient plus sensibles que d'autres à l'influence d'un jugement; 3) la couverture médiatique des jugements aurait un effet d'amplification à court terme sur le mouvement de l'opinion alors qu'à moyen terme, on assisterait à un tassement de l'opinion et à son retour vers ses positions initiales suite au changement de ton de la couverture médiatique.

La présente étude s'inspire, et dans une certaine mesure se démarque, des travaux recensés, et ce à trois niveaux. Elle repose d'abord sur une analyse en panel et se fonde sur un questionnaire visant à dégager les différents éléments de la structure de l'opinion individuelle sur l'avortement. En ce sens, parce que l'information utilisée aura été davantage taillée sur mesure par rapport à des objectifs de recherche spécifiques et qu'elle ne repose pas essentiellement sur l'exploitation de données secondaires (comme dans l'étude de Barnum), il est permis d'espérer que le mouvement de l'opinion pourra être mesuré avec plus de précision. D'autre part, la présente analyse tentera de tenir la part égale entre les travaux portant sur l'effet des jugements (Barnum) et ceux davantage axés sur l'identification des individus plus susceptibles d'être ébranlés par ceux-ci (Lehne et Reynolds). En somme, deux questions centrales orienteront notre propos: 1) le jugement de la Cour a-t-il eu un impact sur l'opinion publique?; et 2) quel est le profil de ceux qui ont modifié leur opinion par rapport à la question de l'avortement?

Mais avant de présenter notre recherche quasi-expérimentale, examinons d'abord la dynamique générale cernée par les enquêtes d'opinion habituelles. 


\section{Des données insuffisantes}

Comment l'opinion publique canadienne a-t-elle fluctué sur la question de l'avortement et plus particulièrement depuis le jugement de la Cour suprême du Canada? Pour répondre à cette question, nous disposons des données de la maison Gallup reproduites au tableau 1. Ces données permettent de retracer l'évolution de l'opinion publique canadienne avant et après le jugement rendu par la Cour suprême en janvier 1988. On y constate qu'avant le jugement de la Cour, soit de 1975 à 1983, la permissivité conditionnelle (c'est-à-dire l'avortement légal dans certains cas) avait déjà subi une fluctuation complète marquée d'abord par une croissance significative de 10 points de pourcentage en 1978 puis par un repli du même ordre en 1983. Ce double déplacement s'est d'abord produit au détriment des positions radicales (c'est-à-dire toujours légal, jamais légal), puis à leur profit. On constate aussi que, peu de temps après le jugement, le déclin de la permissivité conditionnelle observé entre 1978 et 1983, s'est poursuivi pour atteindre $55 \%$ des opinions et ce à l'unique profit de la légalisation inconditionnelle qui atteint alors le plus haut score (28\%) jamais mesuré par la maison Gallup. Toutefois, quatre mois plus tard (juin 1988), se dessine un raffermissement très net de la position majoritaire en faveur de la permissivité conditionnelle aux dépens de la légalisation inconditionnelle, raffermissement qui s'accentue en septembre 1988 pour s'estomper en février 1989. Un an après le jugement de la Cour, l'opinion publique semble donc être revenue à la case départ ou presque.

Il importe toutefois de bien noter que les données de Gallup ne permettent pas d'établir un lien direct entre le jugement de la Cour et les fluctuations de l'opinion. Cela essentiellement pour deux raisons: 1) la mesure d'opinion qui précède le jugement est de beaucoup trop antérieure à celui-ci pour permettre d'en serrer de près l'effet propre; 2) les mesures de l'opinion établies par Gallup proviennent d'échantillons différents de sorte que les fluctuations dans les mesures ne correspondent que bien imparfaitement aux fluctuations dans l'opinion puisque ces dernières devraient, pour qu'on les saisisse dans leur entièreté, être mesurées au niveau individuel et non pas seulement au niveau collectif comme c'est le cas dans les mesures de Gallup. 
Tableau 1: Les attitudes concernant l'avortement au Canada (en pourcentage)

\begin{tabular}{|c|c|c|c|c|c|c|c|}
\hline & \multicolumn{3}{|c|}{ avant le jugement } & \multicolumn{3}{|c|}{ après le jugement } & \multirow[b]{2}{*}{$02 / 89$} \\
\hline & 75 & 78 & 83 & $02 / 88$ & $06 / 88$ & $09 / 88$ & \\
\hline \multirow{5}{*}{$\begin{array}{l}\text { L'avortement } \\
\text { devrait être: } \\
\text { - légal dans tous } \\
\text { les cas } \\
\text { - légal dans } \\
\text { certains cas } \\
\text { - illégal dans } \\
\text { tous les cas } \\
\text { - ne sait pas }\end{array}$} & & & & & & & \\
\hline & 23 & 16 & 23 & 28 & 25 & 20 & 27 \\
\hline & 59 & 69 & 60 & 55 & 59 & 65 & 59 \\
\hline & 17 & 14 & 16 & 13 & 15 & 13 & 13 \\
\hline & 1 & 1 & 1 & 4 & 2 & 2 & 2 \\
\hline
\end{tabular}

Source: The Gallup Report, février et septembre 1988.

Pour tenter de cerner de plus près le jugement de la Cour et le mouvement de l'opinion, il faudrait donc: 1) une mesure de l'opinion qui précède le plus immédiatement possible le jugement de la Cour et 2) une mesure de l'opinion qui permette d'en saisir le mouvement au niveau individuel, ce qui n'est possible qu'à l'aide d'un sondage en panel, où le même échantillon de personnes est interrogé à plusieurs reprises.

\section{La procédure quasi-expérimentale}

Nous avons administré à deux reprises à des étudiants de niveau collégial et universitaire un ensemble de questions se rapportant à la réglementation de l'avortement. On trouvera plus loin les énoncés de ces questions. Tel qu'on peut le constater au tableau 2, notre schéma d'enquête comporte les caractéristiques suivantes: 1) la première vague de questionnaire a eu lieu quelques jours avant le jugement de la Cour, la seconde vague a eu lieu un peu plus de trois semaines après le jugement: 2) 146 personnes ont répondu au questionnaire lors de la première vague et 136 lors de la seconde; 117 répondants ont été présents lors des deux vagues, soit $80 \%$ des 146 répondants originaux. 
Le panel en deux vagues est donc composé de 117 répondants. Étant donné que les panelistes ne se distinguaient pas des individus ayant participé à seulement l'une des deux vagues, il nous a été possible de conclure au caractère aléatoire du phénomène de l'attrition dans notre étude. Le tableau 2 illustre la procédure utilisée.

Tableau 2: Le schéma quasi-expérimental

\begin{tabular}{lccc}
\hline Événement: & Vague 1 & Jugement de la Cour & Vague 2 \\
\hline date: & $25 / 1 / 88$ & $28 / 1 / 88$ & $21 / 2 / 88$ \\
\hline $\mathrm{N}:$ & 146 & & 136 \\
$\mathrm{~N}$ (panélistes): & & &
\end{tabular}

Ce schéma respecte les deux conditions posées plus haut. Il comporte toutefois certaines limites dont il convient de bien cerner la signification. La première a trait au caractère quasi-expérimental de l'enquête qui tient au fait que les sujets n'ont pas été choisis au hasard comme devant représenter une population quelconque. On ne pourra donc pas interpréter nos résultats à la lumière de ceux de la maison Gallup, lesquels sont représentatifs de la population adulte canadienne. L'impact qui nous intéressera ici sera donc exclusivement celui exercé sur certains étudiants, de sorte que notre discours se limitera à explorer comment le jugement de la Cour a influé sur le mouvement de l'opinion chez ces seules personnes. En conséquence, il ne sera pas possible, suite à notre analyse, de généraliser les résultats obtenus à l'ensemble de la population canadienne. Il sera toutefois possible d'étudier comment le changement d'opinion s'est opéré chez les étudiants interrogés et lesquels d'entre eux ont été sujets à changer d'opinion. En conséquence, notre étude permet d'explorer la façon dont l'analyse en panel peut faire ressortir certaines caractéristiques propres au mouvement de l'opinion induit par le jugement de la Cour suprême. L'analyse idéale aurait nécessité soit que Gallup ait procédé par panel, soit que notre propre panel ait été représentatif de toute la population. Même en l'absence de cet idéal toutefois, nos résultats demeurent 
dignes d'intérêt puisqu'ils permettent de saisir, grâce à l'utilisation du panel, diverses modalités de l'impact du jugement de la cour, modalités dont il y a lieu de croire, puisque les étudiants font partie de la population canadienne, qu'elles tiendraient aussi pour cette population mais dans des proportions impossibles à estimer avec les données actuelles.

La seconde limite concerne le nombre relativement faible de répondants dans le panel. Ces deux limites ne sont certes pas absolues mais elles démontrent bien le caractère exploratoire de la recherche.

\section{La description: mesure du mouvement}

Pour analyser la mouvance de l'opinion, nous ne nous sommes pas limités à la simple question habituellement posée dans les sondages sur l'avortement. Le questionnaire visait à articuler plusieurs composantes de l'opinion à travers un ensemble de questions semblables à celles utilisées par Marsh (1985):

- En principe, croyez-vous que l'avortement devrait être ... accessible dans tous les cas? ... accessible seulement dans certains cas... inaccessible dans tous les cas?

- Selon vous, la loi canadienne actuelle permet-elle l'avortement... dans tous les cas? ... seulement dans certains cas?... dans aucun cas?

- Selon vous, la majorité des gens veulent-ils que la loi sur l'avortement... demeure inchangée?... soit amendée pour rendre l'avortement plus accessible? ... soit amendée pour rendre l'avortement moins accessible?

- Croyez-vous que, dans l'avenir, l'opinion publique relativement à l'avortement aura tendance ... à demeurer à peu près stable? ... à se déplacer en faveur d'un élargissement de l'accessibilité à l'avortement? ... à se déplacer en faveur d'une restriction de l'accessibilité à l'avortement?

- Vous-même, pensez-vous que la loi sur l'avortement devrait ... demeurer inchangée? ... être amendée pour rendre l'avortement plus accessible? ... être amendée pour rendre l'avortement moins accessible? 
- Diriez-vous que votre opinion concernant l'opportunité de modifier la loi sur l'avortement ... est à peu près définitivement arrêtée? ... est provisoirement arrêtée? ... n'est pas arrêtée?

Les questions ont pour objet de mesurer: le principe, l'interprétation de la loi, la position quant à la loi, la perception de l'état de l'opinion publique, la perception de la tendance de l'opinion publique et la fixité de l'opinion individuelle.

Ces différentes variables ont été construites à partir de la double hypothèse suivante: 1) il existe une relation entre les composantes de l'opinion individuelle; et 2) la dynamique des dites composantes n'est pas étrangère à la mouvance de l'opinion.

Les résultats agrégés

Par rapport à ces différentes composantes de l'opinion, la mesure du mouvement peut être analysée sous deux angles: soit au niveau collectif ou agrégé, soit au niveau individuel ou désagrégé. Les tableaux 3 et 4 fournissent les principaux résultats collectifs. On y trouve d'abord les résultats pour chacune des deux vagues puis la différence calculée sur la base de l'échantillon global suivant la procédure utilisée par Marsh dans son enquête.

\section{Tablau 3: Les résultats collectifs (en pourcentage)}

\begin{tabular}{|c|c|c|c|c|c|c|c|c|}
\hline & \multicolumn{2}{|c|}{ Principe* } & \multicolumn{3}{|c|}{ Interprétation de la loi } & \multicolumn{3}{|c|}{ Position sur la loi } \\
\hline & favor. & défavor. & Tous & Certains & Aucun & +Acces. & Inch & Acces. \\
\hline $\begin{array}{l}\text { Vague I } \\
\text { Vague II } \\
\text { Différence }\end{array}$ & $\begin{array}{r}50,0 \\
52,6 \\
+2,6\end{array}$ & $\begin{array}{l}50,0 \\
47,4 \\
-2,6\end{array}$ & $\begin{array}{r}27,4 \\
59,8 \\
+32,4\end{array}$ & $\begin{array}{r}64,1 \\
39,3 \\
-24,8\end{array}$ & $\begin{array}{r}8,5 \\
0,9 \\
=7,6\end{array}$ & $\begin{array}{l}65,8 \\
59,8 \\
-6,0\end{array}$ & $\begin{array}{l}21,4 \\
27,4 \\
+6,0\end{array}$ & $\begin{array}{r}12,8 \\
12,8 \\
0,0\end{array}$ \\
\hline
\end{tabular}

* La variable principe a été ramenée à deux catégories distinguant les individus favorables ou défavorables à la permissivité radicale (l'avortement accessible dans tous les cas).

Ce tableau indique en tout premier lieu que la majorité des répondants estiment que la loi doit rendre l'avortement plus accessible. Dans notre échantillon peu d'individus optent pour une position 
conservatrice. Deuxièmement, et c'en est là tout l'intérêt, le tableau indique des déplacements inégaux entre les deux vagues: faibles pour le principe; très marqués pour l'interprétation de la loi et intermédiaires pour la position. Les répondants ont modifié fortement leur interprétation de la loi sans pour autant modifier leur position. Il n'y a pas uniformité dans les mouvements. Ces résultats indiquent aussi que collectivement les individus n'ont pas réellement, suite au jugement de la Cour, modifié leur principe par rapport à la question de l'avortement. Le tableau 4 fournit les données propres à la perception de l'opinion d'autrui.

Tableau 4: Les résultats collectifs, la perception d'autrui (en pourcentage)

\begin{tabular}{lrrrrrr}
\hline & \multicolumn{3}{c}{ Perception de l'état } & \multicolumn{3}{c}{ Perception de la tendance } \\
& +Acces. & Inch & -Acces. & +Acces. & Inch & -Acces. \\
\hline Vague I & 17,9 & 53,0 & 29,1 & 50,9 & 30,2 & 19,0 \\
Vague II & 27,4 & 53,0 & 19,7 & 59,5 & 30,2 & 10,2 \\
Différence & $+9,5$ & 0,0 & $-9,4$ & $+8,6$ & 0,0 & $-8,7$ \\
\hline
\end{tabular}

Ce tableau contient des données pertinentes sous deux angles. Premièrement, nos répondants ayant opté pour une plus grande accessibilité ne perçoivent pas l'opinion publique comme nécessairement convergente à la leur, tout au moins dans le présent. La convergence est plutôt posée dans l'avenir. Si le présent ne nous donne pas raison ... l'histoire prouvera la justesse de notre position, se disent-ils en somme. Et le jugement de la Cour renforce cette conviction déjà présente lors de la première vague. Deuxièmement, le tableau 4 indique - lorsqu'on le compare au tableau 3 - que si collectivement les individus modifient peu leur principe et leur position, leur perception de la position d'autrui est sujette à plus de mobilité. Qui plus est, cette mobilité va dans le sens du jugement rendu. Ainsi, après celui-ci, deux déplacements de l'opinion se manifestent: d'une part, et de façon massive, la loi est réinterprétée dans un sens plus permissif, d'autre part, et c'est ici que se pose véritablement la question de l'effet d'entraînement de la décision de la 
Cour, l'opinion agrégée est perçue comme étant plus favorable à la thèse d'une plus grande accessibilité à l'avortement. Or, si l'individu est sensible à l'état et à la tendance de l'opinion collective dans son processus de positionnement sur une question donnée (Lazarfeld, Berelson et Gaudet, 1948; Berelson, Lazarfeld et McPhee, 1962; Marsh, 1984, 1985, Lang et Lang, 1984), il en résulterait qu'en modifiant sa perception de l'opinion majoritaire, l'individu serait amené à modifier son opinion individuelle. L'ampleur de cet effet indirect du jugement de la Cour suprême sera examiné plus loin.

\section{Les résultats désagrégés}

Les résultats agrégés, s'ils fournissent une première image, demeurent insuffisants. Ils masquent une dynamique se produisant à une autre échelle. En fait, lorsque nous procédons à l'analyse des déplacements des répondants eux-mêmes, la mouvance est beaucoup plus marquée. Les résultats désagrégés nous founissent un matériel en fait plus riche.

Prenons comme point de départ la question de principe. Même si, à la marge, les résultats sont quasi-identiques d'une vague à l'autre $(2,6 \%)$ - nous comptons 21 individus qui ont opéré un déplacement, soit $18,4 \%$. Les déplacements individuels sont ici 6 fois plus importants que les résultats à la marge. Les résultats individuels, puisqu'ils sont contradictoires et s'annulent de ce fait, ne sont pas visibles à la marge. Pour l'interprétation de la loi, 55 répondants ont modifié leur choix soit $37,0 \%$. Enfin, $35,8 \%$ des répondants ont modifié leur position. Une fois de plus, il n'y a donc pas uniformité dans les déplacements des individus. Le tableau 5 désagrège complètement les mouvements des individus. Il fournit une image exhaustive des déplacements individuels des répondants sur le principe, l'interprétation de la loi et la position. Il permet en somme de visualiser dans quel sens les déplacements s'opèrent. La lecture peut se faire ainsi. Lors de la première vague, 57 individus étaient, sur la question de principe, favorables à l'accessibilité complète. De ceux-ci, 9 se sont déplacés lors de la deuxième vague vers la case défavorable à cette accessibilité complète; 12 individus ont effectué un mouvement 
inverse. La flèche indique donc le sens du déplacement. Il faut souligner ici que si les résultats agrégés ou à la marge s'appuient sur la différence $(12-9=3,3 / 114=2,6 \%)$ les résultats désagrégés découlent de la sommation des déplacements $(12+9=211,21 / 114=18,4 \%)$. L'écart heuristique entre les deux modes d'exploration du mouvement est manifeste ici.

\section{Tableau 5: La mobilité désagrégée}

Mobilité individuelle par rapport au principe

\begin{tabular}{|c|c|c|c|c|c|}
\hline \multirow{3}{*}{ Vague I } & \multicolumn{3}{|c|}{ Vague II } & \multirow{2}{*}{57} & \multirow[b]{2}{*}{$(50,0 \%)$} \\
\hline & Favorable & $\begin{array}{c}\text { Favorable } \\
48 *\end{array}$ & $\stackrel{\text { Défavorable }}{\longrightarrow} 9$ & & \\
\hline & Défavorable & $\begin{array}{l}12 \leftarrow \\
60 \\
(52,6 \%)\end{array}$ & $\begin{array}{l}-45 \\
54 \\
(47,4 \%)\end{array}$ & $\begin{array}{l}57 \\
114^{\text {** }}\end{array}$ & $\begin{array}{r}(50,0 \%) \\
(100.0 \%)\end{array}$ \\
\hline
\end{tabular}

Mobilité individuelle par rapport à l'interprétation de la loi

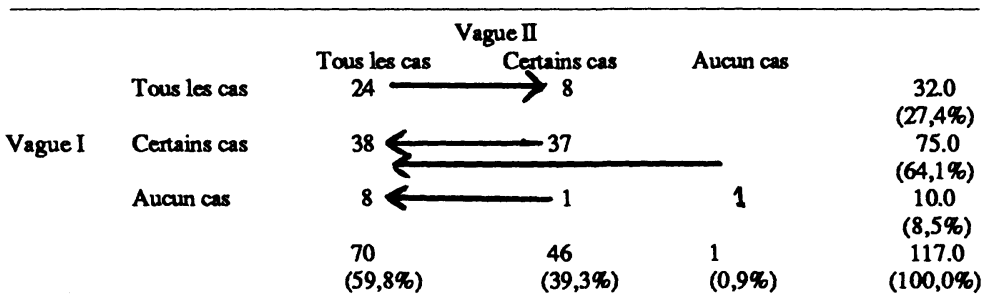

Mobilité individuelle par rapport à la position

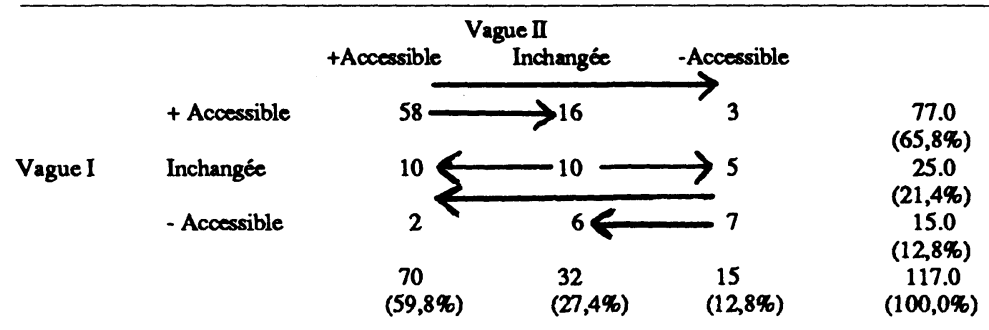

* Absence de mouvement .** 3 répondants n'ont pas répondu à la question sur le principe lors d'une des deux vagues. 
Le tableau 6 présente les pourcentages relatifs aux deux types de changement:

Tableau 6: Mouvance collective et individuelle

\begin{tabular}{lcc}
\hline & $\begin{array}{c}\% \text { de mouvance } \\
\text { collective }\end{array}$ & $\begin{array}{c}\% \text { de mouvance } \\
\text { individuelle }\end{array}$ \\
\hline Principe & 2,6 & 18,4 \\
Position & 6,0 & 35,8 \\
Interprétation & 32,4 & 47,0 \\
Perception (état) & 9,5 & 47,0 \\
Perception (tendance) & 8,6 & 47,0 \\
\hline
\end{tabular}

La cohérence et l'incohérence

On notera, à la lecture du tableau 5, l'impact qu'a eu le jugement de la Cour sur l'interprétation de la loi: 62 personnes $(53,0 \%)$ n'ont pas changé leur interprétation, $47(40,2 \%)$ l'ont interprétée de façon plus permissive et $(6,8 \%)$ de façon plus restrictive. Cela signifie que la loi, à propos de laquelle les individus ont pris position, a vu son interprétation être modifiée de façon différente selon les individus. Peu importe que ces modifications d'interprétation soient ou non légalement fondées, elles permettent d'établir avec précision non seulement la signification de la loi à chacune des vagues mais aussi comment les changements dans l'interprétation de la loi entraînent des changements dans la position vis-à-vis cette loi, ce que nous examinerons maintenant conjointement avec les changements sur le principe, sous l'appellation de cohérence. En effet, le mouvement désagrégé nous permet d'identifier la cohérence des individus à un moment donné et la cohérence des mouvements qu'ils effectuent sur la position, le principe, et l'interprétation de la loi. Présentons d'abord les différentes figures d'incohérence qui combinent cette fois trois variables: le principe, l'interprétation de la loi et la position. Par exemple, un individu qui croit que la loi permet l'avortement dans tous les cas, et qui, au plan des principes est favorable à la permissivité 
radicale doit opter pour la position inchangée. S'il croit au contraire que la loi doit être plus accessible il entre en contradiction avec luimême; en ce sens il est identifié comme «incohérent». L'incohérence, telle que définie, ne doit pas être mésinterprétée. Elle ne renvoie pas à l'opposition qui peut en toute logique et en toute cohérence exister entre le refus de l'avortement pour soi et son support du point de vue collectif, puisqu'aucune question n'était de l'ordre du choix individuel. L'opinion est ici considérée uniquement du point de vue collectif, par rapport à une accessibilité pour les autres, suivant le libellé des différentes questions.

Le tableau 7 présente les 18 possibilités construites à partir des trois variables et les résultats.

\section{Tableau 7: L'incohérence théorique, vagues I et II}

L'incohérence, théorique

\begin{tabular}{lcccccc}
\hline & \multicolumn{8}{c}{ Principe et interprétation de la loi } & & \\
Position & F-T & F-C & F-A & D-T & D-C & D-A \\
+ Accessible & I & C & C & I & C & C \\
Inchangé & C & I & I & I & C & C \\
- Accessible & I & I & I & C & C & I
\end{tabular}

L'incohérence: Vague I

\begin{tabular}{lccccccc}
\hline & \multicolumn{7}{c}{ Principe et interprétation de la loi } \\
Position & F-T & F-C & F-A & D-T & D-C & D-A \\
+ Accessible & 4 & 39 & 3 & 6 & 21 & 4 \\
Inchangée & 9 & 1 & 0 & 5 & 9 & 1 \\
-Accessible & 0 & 1 & 1 & 8 & 4 & 1
\end{tabular}

Répartition des incohérents: Vague II

\begin{tabular}{|c|c|c|c|c|c|c|}
\hline \multirow{5}{*}{$\begin{array}{l}\text { Position } \\
+ \text { Accessible } \\
\text { Inchangée } \\
\text { - Accessible }\end{array}$} & \multicolumn{4}{|c|}{ Principe et interprétation de la loi } & \multirow{5}{*}{$\begin{array}{l}\text { D-C } \\
13 \\
6 \\
0\end{array}$} & \multirow{5}{*}{$\begin{array}{c}\text { D-A } \\
1 \\
0 \\
0\end{array}$} \\
\hline & F-T & F-C & F-A & D-T & & \\
\hline & 18 & 26 & 0 & 12 & & \\
\hline & 15 & $\mathbf{0}$ & $\mathbf{0}$ & 9 & & \\
\hline & $\mathbf{1}$ & o & $\mathbf{0}$ & 13 & & \\
\hline
\end{tabular}


Les résultats - établis à partir des figures théoriques d'incohérence - sont très éloquents. Trois points doivent être ici dégagés. Premièrement, lors de la vague initiale $16,7 \%$ (19/114) des individus furent incohérents. Lors de la seconde vague, l'incohérence atteint $35,1 \%(40 / 114)$ soit plus du double. De la première à la seconde vague il y a donc montée de l'incohérence.

Deuxièmement, 91,5\% (54 sur 59) des incohérents se retrouvent dans trois cases qui ont pour point commun d'interpréter la loi suivant une optique très libérale, c'est-à-dire rendant l'avortement possible dans tous les cas. Or comme la case qui combine cette dernière interprétation et une position prônant une plus grande accessibilité est identifiée comme une case d'incohérence, il est compréhensible - étant donné la distribution initiale de notre échantillon - qu'un grand nombre d'individus ait commis ce pléonasme.

Le troisième point enfin découle des deux précédents. Compte tenu de l'augmentation du nombre d'incohérents entre les deux vagues et des cases d'où cette croissance provient, la Cour suprême, plutôt que de clarifier les options s'est donc trouvée à créer une confusion par rapport aux différentes options. En fait, les individus ont ainsi modifié leur interprétation de la loi, sans par ailleurs modifier du même coup leur position sur celle-ci. Il semble, en fait, y avoir décalage entre les deux mouvements.

Le manque d'uniformité dans les déplacements collectifs et individuels sur les variables-clés de l'opinion prend nettement forme à travers la montée de l'incohérence. Celle-ci est la preuve que le mouvement des individus est asynchronique. Voilà pour ce qui est de la description. Essayons à présent d'expliquer le phénomène de l'asynchronie, visible avec précision dans les résultats désagrégés mais également manifeste au niveau des résultats agrégés.

\section{L'explication: les facteurs du mouvement}

Pourquoi certains répondants ont-ils modifié leur opinion et d'autres non? Existe-t-il des profils de répondants susceptibles d'expliquer leur mouvement? C'est à cette question que cette seconde 
partie tente de répondre. Le mouvement étant à présent décrit, il importe de l'expliquer. Il s'agit de montrer que cette mobilité n'est pas aléatoire. Deux types de facteurs doivent retenir notre attention: les facteurs statiques tels que mesurés lors de la première vague et les facteurs dynamiques ressortissants de changements survenus entre les deux vagues.

\section{Les facteurs statiques}

1) La fixité. D'une manière évidente, la fixité que les répondants s'attribuent a une incidence sur la mouvance. La preuve est simple: le pourcentage de mouvants augmente dans la mesure où les répondants considèrent que leur opinion n'est pas arrêtée: $23,8 \%$ de mouvants chez ceux qui considèrent que leur opinion est «définitivement arrêtée»; $35,9 \%$ chez ceux qui considèrent que leur opinion est «provisoirement arrêtée» et $50 \%$ de mouvants chez ceux qui considèrent que leur opinion n'est «pas arrêtée».

2) La position. La case où se trouve au départ les individus constitue également une cause de changement; les individus qui ont adopté, lors de la première vague, la position «inchangée» et «moins accessible» ont changé de position dans une proportion de $57,5 \%$. Les individus ayant au contraire opté pour la case «plus accessible» ont changé de position dans une proportion de $24,6 \%$ seulement, soit moins de la moitié. En somme, les positions qui se sont vues contredites par le jugement ont donné lieu à plus de mouvance. C'est là un deuxième élément. Toutes les cases ne donnent donc pas lieu à une mouvance identique.

3) La cohérence. Si l'incohérence constitue l'une des facettes du mouvement, elle peut également être considérée comme une cause. Les individus reconnus «incohérents» lors de la première vague ont été plus mobiles que les répondants considérés «cohérents», alors que $34,4 \%$ des répondants réputés «cohérents» lors de la première vague ont changé de position, $44,4 \%$ des répondants «incohérents» ont modifié leur position. En ce sens, l'incohérence, telle que décrite, constitue un autre facteur de mouvement. 
La fixité, la position et la cohérence sont les trois facteurs statiques qui expliquent le mouvement de nos répondants. Celui-ci n'est donc pas simplement aléatoire. L'explication du mouvement renvoie à un profil, ou tout au moins à des caractéristiques données, existantes lors du premier coup de sonde.

\section{Facteurs dynamiques}

Mais il existe aussi d'autres facteurs, que nous nommons dynamiques parce qu'ils se mesurent entre la vague I et la vague II, donc conjointement au mouvement que nous tentons de circonscrire. Deux facteurs dynamiques doivent être étudiés: la propension au mouvement et la direction.

1) La propension au mouvement. Nos résultats montrent en premier lieu que plus les répondants modifient leur opinion sur le principe, l'interprétation de la loi, et les perceptions de l'état et de la tendance, plus grande est la propension à changer de position. Il y aurait ainsi une relation entre la mouvance de la position et la mouvance des éléments constitutifs de cette position. Deux éléments confirment cette relation. Premièrement, parmi les individus qui ont modifié leur opinion sur deux des éléments constitutifs cités ou plus, $51,6 \%$ ont modifié leur position. À l'opposé, seulement $11,8 \%$ des individus qui n'ont effectué aucun changement ont modifié leur opinion.

2) La direction du mouvement. Pour expliquer le mouvement, nous avons cherché à identifier bien plus qu'une position ou qu'un changement de position mais aussi une direction au déplacement: ainsi, au niveau de l'interprétation de la loi, effectuer le mouvement de "aucun cas» à "certains cas» est semblable au mouvement qui consiste à passer de "certains cas» à «tous les cas» dans la mesure où l'on considère que ces deux mouvements ont un point en commun, soit la direction plus permissive quant à l'interprétation de la loi. Cette même logique s'applique également à la position sur la loi. De ce point de vue, on peut aussi établir la direction d'un changement de position selon que l'individu modifie 
celle-ci dans un sens plus restrictif, plus permissif ou qu'il opte pour le statu quo.

Tableau 8: Concordance entre les changements individuels relatifs à l'interprétation de la loi, la perception de la tendance de l'opinion publique et la position sur la loi

8a) Concordance entre les changements individuels relatifs à l'interprétation de la loi et la position sur la loi concernant l'avortement

Direction du changement sur l'interprétation de la loi (\%)

$\begin{array}{llccc} & & + \text { Permissif } & \text { Aucun } & \text { +Restrictif } \\ \text { Direction du } & + \text { Permissif } & 12,8 & 11,3 & 62,5 \\ \text { changement } & \text { Aucun } & 51,8 & 77,4 & 37,5 \\ \text { sur la position } & + \text { Restrictif } & 36,2 & 11,3 & 0,0 \\ & & 100,0 & 100,0 & 100,0\end{array}$

8b) Concordance entre les changements individuels relatifs à la tendance de l'opinion publique et la position sur la loi concernant l'avortement

Direction du changement sur la perception de la tendance de l'opinion publique (\%)

$\begin{array}{llccc} & & + \text { Permissif } & \text { Aucun } & \text { + Restrictif } \\ \text { Direction du } & + \text { Permissif } & 34,3 & 9,8 & 0,0 \\ \text { changement } & \text { Aucun } & 60,8 & 68,9 & 55,0 \\ \text { sur la position } & + \text { Restrictif } & 5,7 & 21,3 & 45,0 \\ & & 100,0 & 100,0 & 100,0 \\ & & & \end{array}$

L'étape suivante a consisté à examiner la concordance de ces mouvements, et plus particulièrement deux concordances. Premièrement celle entre le changement directionnel sur la position et le changement directionnel sur l'interprétation de la loi: à titre d'exemple, si un répondant, n'ayant pas modifié son choix sur le principe (soit le plus grand nombre des répondants: $81,6 \%$ ), modifie entre les deux vagues son interprétation de la loi dans un sens plus permissif, le mouvement attendu sur la position sera restrictif. La deuxième concordance est celle entre les changements directionnels dans la perception de la tendance de l'opinion collective et les 
changements directionnels sur la position. Un individu va-t-il se mouvoir en harmonie avec la direction du changement qu'il reconnaît autour de lui? Tel est ici le problème. Les tableaux 8a) et $8 b$ ) présentent les données de ces deux concordances.

En ce qui concerne le lien entre changements directionnels dans l'interprétation de la loi et de la position, trois résultats doivent être signalés:

1) Il apparaît d'abord clairement que le fait de modifier son interprétation de la loi a été un facteur décisif de changement de l'opinion individuelle puisque, parmi ceux qui n'ont pas modifié cette interprétation, $77,4 \%$ ont maintenu leur position d'une vague à l'autre alors que ces pourcentages chutent respectivement à $51,8 \%$ et à $37,5 \%$ chez les individus ayant changé leur interprétation de la loi dans un sens permissif ou restrictif.

2) De façon générale, très peu de changements a contrario se sont manifestés (aucun chez ceux qui avaient modifié leur interprétation de la loi dans un sens restrictif; ils sont par ailleurs trois fois moins nombreux que les changements cohérents chez les répondants ayant changé leur interprétation de la loi dans un sens plus permissif).

3) On remarque enfin la relative réticence des individus ayant changé leur interprétation de la loi dans un sens permissif (contingent important de répondants ainsi que l'atteste le tableau 5) à modifier leur position dans un sens restrictif puisque $51,8 \%$ de ceux-ci n'effectuent aucun changement sur la position. Cela explique la monté de l'incohérence entre les deux vagues (voir le tableau 7).

L'examen de la relation entre les variations de la perception de la tendance de l'opinion collective et de la position individuelle est également instructif (voir le tableau 8b). Dans la mesure où la décision de la Cour suprême a provoqué un déplacement plus généralisé des perceptions allant dans le sens d'un plus grand libéralisme (voir les tableaux 4 et 5), il n'est pas interdit de penser que l'asynchronisme, la montée de l'incohérence dont nous avons traité précédemment, trouve là une partie de son explication dans une interaction complexe mettant en action des changements vers la permissivité tant en ce qui concerne l'interprétation de la loi que la perception de la tendance (un individu cumulant ces deux changements serait en butte à des pressions 
contradictoires, le changement dans l'interprétation de la loi l'amenant à modifier sa position dans un sens restrictif, le changement dans la perception de la tendance le poussant dans le sens contraire).

\section{Conclusion}

Évaluons enfin les apports et les limites de cette étude exploratoire, en commençant par les résultats ou les apports.

1. Au plan de la méthodologie générale, notre étude démontre clairement que l'analyse des résultats individuels est considérablement plus riche en enseignements que ne l'est l'analyse à la marge. Lorsqu'on se limite à cette dernière, l'impact de la Cour est masqué.

2. Le jugement de la Cour suprême a eu un effet complexe sur les individus: ceux-ci n'ont pas modifié d'une manière logiquement organisée les différentes composantes de leur opinion. Les individus réagissent d'une manière asynchronique par rapport aux composantes internes de leur opinion. L'interprétation de la loi est d'abord modifiée sans que les autres aspects ne soient modifiés du même coup. Entre la première et la deuxième vague, les individus 1) ont peu modifié leur principe, 2) ont massivement changé leur interprétation de la loi, 3) ont modifié leur perception (état, tendance) dans le sens de la permissivité, 4) n'ont pas modifié leur position sur la loi de façon aussi importante que ne l'annonçait leur changement dans l'interprétation de la loi.

3. Cet asynchronisme se manifeste par les variations inégales des différentes variables - aussi bien individuellement que collectivement - et par la montée de l'incohérence.

4. Cet asynchronisme s'explique par des facteurs statiques et par des facteurs dynamiques. Les individus subissent d'autant plus l'impact de la Cour, en modifiant leur position, qu'ils: 1) se disaient «peu fixés»; 2) optaient pour une position contraire à celle émise par la Cour; 3) furent incohérents lors de la première vague. D'une manière dynamique, 1) plus les individus bougent sur d'autres variables, plus ils seront portés à changer de position; 2) plus ils modifient leur interprétation de la loi dans une direction donnée, plus du même coup ils - c'est-à-dire la majorité — avaient tendance à effectuer un 
mouvement sur la position pour maintenir une accessibilité équivalente; et 3) plus ils changent leur perception de la tendance dans la direction de la permissivité, plus ils ont tendance à changer de position dans le même sens.

5. Théoriquement, notre étude s'inscrit dans un réseau complexe. Premièrement, sans contredire les idées avancées par Baas et Thomas, notre recherche démontre que la Cour a eu un impact sur les individus et que cet impact, loin d'être aléatoire, semble s'inscrire dans le sens du jugement prononcé par la Cour suprême. D'une manière quasi-expérimentale, notre recherche confirme plutôt les idées avancées par Dahl mais avec une force variable: faible sur le principe et forte sur la perception de l'opinion individuelle; à la limite, la Cour ne semble pas avoir légitimé d'une manière nette la cause des partisans du libre choix. Son effet est très fort précisément là où se trouve la fonction de la Cour, soit au niveau de l'interprétation de la loi. Et à partir de ce point, la Cour a bousculé à court terme la cohérence relative des individus; son incidence n'est donc pas directe mais se manifeste via l'obligation de repenser le problème (Goettsh, 1983) dans la mesure où elle introduit de nouvelles données dans les schémas cognitifs établis et force un réaménagement de ceux-ci. La question de la cohérence, deuxièmement, rejoint aussi ce qui a été avancé par quelques auteurs: la majorité des individus auraient des schèmes cognitifs peu organisés ou faiblement structurés (Navarro, C., 1982; Lodge et Hamil, 1986; Rosenberg, S.W., 1988). Une modification des paramètres juridiques sur une question aurait ainsi des répercussions non-synchronisées dans la mesure où l'ensemble des schèmes cognitifs ne sont pas nécessairement liés logiquement. Notre travail recoupe enfin les travaux de chercheurs ayant travaillé le rapport entre la position individuelle et la perception du mouvement collectif de l'opinion (Noelle-Neuman, 1984; Marsh, 1985; Lang et Lang, 1984; Lazarfeld et al., 1948). La direction de la causalité est ici impossible à définir. Le problème reste entier sur ce point.

En somme, notre étude montre la complexité des processus de réception des stimuli, dans ce cas le jugement de la Cour suprême. La Cour a exercé un effet mais les individus ne réagissent ni uniformément, ni mécaniquement. Il y a donc deux niveaux de causalité: celui de la Cour et les caractéristiques individuelles. Par 
ailleurs, nos résultats et nos outils conceptuels montrent qu'il est possible de décrire et d'expliquer, partiellement du moins, la complexité du mouvement. Tel est l'apport général de cette recherche exploratoire. Toutefois, si celle-ci nous permet de mieux comprendre la dynamique de la mouvance, elle contient des limites importantes.

1. La première est sans aucun doute le petit nombre de répondants. Rappelons que toute cette recherche est fondée sur 117 répondants. Dans la mesure où l'exercice a une vocation exploratoire, ce nombre réduit n'invalide aucunement le sens de l'entreprise. Toutefois, certains résultats ont une portée limitée dans la mesure où peu d'individus y ont été associées.

2. La deuxième limite n'est point de notre ressort; elle relève de l'événement lui-même. Lorsque les juges se sont prononcés, ils ont créé, en fait un vide juridique. L'ambiguiité était complète. L'article du Code criminel s'est trouvé invalidé; en ce sens, la loi (telle qu'elle existait) a perdu toutes ses dents. Toutefois, en réaction au jugement, tous les intervenants faisaient part de la nécessité d'une nouvelle loi et prenaient position en fonction d'une loi hypothétique. Or, nos répondants ont très bien pu répondre à la question sur l'interprétation de la loi en se référant à la loi - telle que jugée par la Cour puisque seule cette loi avait eu une existence, et prendre position sur la modification de la loi, lors de la deuxième vague, en fonction de ce que devrait être la nouvelle loi, - la loi hypothétique - sans se référer à la loi mise en cause par le plus haut tribunal. L'incohérence croissante et l'asynchronie dans les déplacements s'expliquent peut-être ainsi.

3. La troisième limite renvoie également à l'environnement. Le jugement de la Cour ne peut être considéré comme le seul stimulus reçu par les individus. Au cours des jours qui ont suivi, le gouvernement fédéral, les partis de l'opposition, les gouvernements provinciaux, et les groupes de pression concernés ont pris position eux aussi et ce, dans des directions opposées. Nos répondants ont reçu en fait une avalanche de stimulations sur la question dont le point de départ, seulement, fut le jugement de la Cour. Nous pouvons même affirmer qu'au moment où ils répondaient à notre questionnaire, ils subissaient encore l'influence d'une stimulation multiple et contradictoire. Le temps ne se découpe donc plus aussi simplement que nous le prétendions au départ - l'avant et l'après. Cette limite, si elle 
nous oblige à la nuance, possède un aspect positif. La stimulation sociale est peut-être plus souvent de cet ordre, continue, complexe et contradictoire, que simple ou unidirectionnelle. 


\section{Bibliographie}

BAAS, L., THOMAS, D., «The Supreme Court and Policy of Legitimation: Experimental Tests», American Politics Quaterly, 1984, 12, 335-360.

BARNUM, D.G., «The Supreme Court and Public Opinion: Judicial Decision-Making in the Post New-Deal Period», Journal of Politics, 1985, 47, 652-666.

BERELSON, B., LAZARFELD, P., McPHEE, W.N., Voting, Chicago, University of Chicago Press, 1962.

BRENT, E., GRANDBERG, D., "When Prophecy Bends: the Preference-Expectation Link in US Presidential Elections», Journal of Personality and Social Psychology, 1983, 45, 3, 477-491.

CASEY, G., «Popular Perception of Supreme Court Rulings», American Politics Quaterly, 1976, 4, 3-45.

CLOUTIER, É., GUAY, J., NADEAU, R., «Bandwagoning and Underdoging: a Quasi-Experimental Model of Opinion Movement on Abortion», Groupe de recherche sur l'opinion publique, Université de Montreal.

CLOUTIER, É., GUAY, J., NADEAU, R., «Bandwagoning and Underdoging: a Quasi-Experimental Panel Study of Opinion Movement», paraîtra dans un prochain numéro de l'International Journal of Public Opinion Research.

GALLUP REPORT, divers numéros.

GOETTSCH, S.L., Attitude Extremism in the Abortion Controversy: a Test of Social Judgment, Cognitive Dissonance, and Attribution Theories, These, Iowa State University, 1983, département de sociologie et anthropologie.

LAZARFELD, P., BERELSON, B., GAUDET, H., How People Vote, Columbia, University of Columbia Press, 1948.

LEHNE, R., REYNOLDS, J., «The Impact of Judicial Activism on Public Opinion», American Journal of Political Science, 1978, 896-904.

LODGE, M., HAMILL, R., "A Partisan Schema for Political Information Processing», American Political Science Review, $80,2,1986,503-519$. 
MARSH, C., «Back on the Bandwagon», British Journal of Political Science, (15) 1, 1985, 51-74.

MARSH, C., «Do Polls Affect What People Think?», in C.F. TURNER and E. MARTIN (ED.) Surveying Subjective Phenomena, vol. 2, 565-591 (Sage, 1984).

NAVARRO, C., «Théorie opératoire de l'intelligence et analyse des processus cognitifs de l'adulte dans la réalistion des tâches», 173-193, Perspectives piagétiennes, Toulouse, Privat, 1982.

NISBETT, R. E., et al., «The False Consensus Effects: an Egocentric Bias in Social Perception and Attribution Process», Journal of Experimental Social Psychology, 1977, 13, 279-301.

NOELLE-NEUMAN, E., The Spiral of Silence, Chicago, University of Chicago Press, 1984.

ROSENBERG, S. W., "The Structure of Political Thinking», American Journal of Political Science, 1988, 32, 539-566. 